\title{
Near Transform-Limited Quantum Dot Linewidths in a Broadband Photonic Crystal Waveguide
}

\author{
Freja T. Pedersen, Ying Wang, Cecilie T. Olesen, Sven Scholz, Andreas D. Wieck, Arne Ludwig, \\ Matthias C. Löbl, Richard J. Warburton, Leonardo Midolo, Ravitej Uppu,* and Peter Lodahl*
}

Cite This: ACS Photonics 2020, 7, 2343-2349

Read Online

ACCESS 1

山lll Metrics \& More

回国 Article Recommendations

ABSTRACT: Planar nanophotonic structures enable broadband, near-unity coupling of emission from quantum dots embedded within, thereby realizing ideal single-photon sources. The efficiency and coherence of the single-photon source is limited by charge noise, which results in the broadening of the emission spectrum. We report suppression of the noise by fabricating photonic crystal waveguides in a gallium arsenide membrane containing quantum dots embedded in a $p$-i-n diode. Local electrical contacts in the vicinity of the waveguides minimize the leakage current and allow
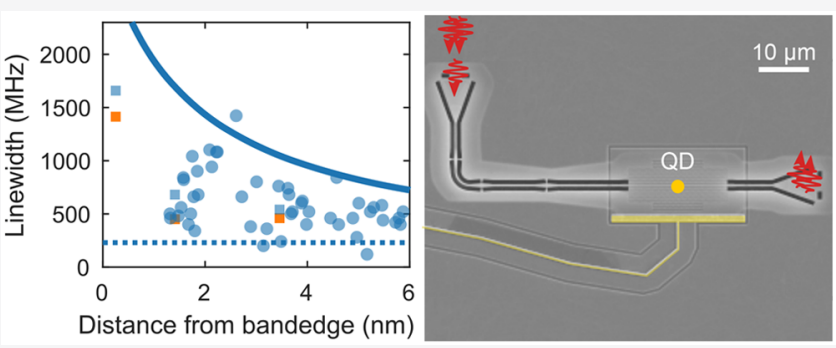
fast electrical control $(\approx 4 \mathrm{MHz}$ bandwidth) of the quantum dot resonances. Resonant linewidth measurements of 51 quantum dots coupled to the photonic crystal waveguides exhibit near transform-limited emission over a $6 \mathrm{~nm}$ wide range of emission wavelengths. Importantly, the local electrical contacts allow independent tuning of multiple quantum dots on the same chip, which together with the transform-limited emission are key components in realizing multiemitter-based quantum information processing.

KEYWORDS: photonic crystal waveguide, quantum dot, single photons, resonant spectroscopy, nanophotonics, semiconductor heterostructure

A on-demand source of indistinguishable single photons is a key building block in a scalable quantum network. ${ }^{1}$ Achieving on-demand operation requires high quantum efficiency of the emitter together with deterministic coupling to a single propagating mode for efficient extraction. Semiconductor quantum dots (QDs) coupled to nanophotonic structures have over the past decade proven to be strong candidates for such a source. $^{2-7}$ In particular, photonic crystal waveguides (PCWs) enable near-unity coupling of the QD emission to a single propagating mode ${ }^{8}$ that can be efficiently extracted across a broad spectral range.

The semiconductor environment of the QD usually introduces fluctuations in the form of charge noise, which leads to a decrease of coherence. ${ }^{9}$ The charge noise stems from variations in the electronic states around the QD, which leads to fluctuations in the local electric field. These changes shift the QD emission energy through the Stark effect and result in a broadening of the emission line. Consequently, the optical linewidth increases significantly above the transform limit determined by the spontaneous emission rate. ${ }^{10}$ Charge noise can be suppressed by embedding the QDs in a diode heterostructure. $^{11}$ So far, transform-limited QDs have been reported in bulk samples, ${ }^{12}$ microcavities, ${ }^{13}$ and in a multimode nanobeam waveguide. ${ }^{14}$ However, in many cases, broadband approaches featuring efficient photon-emitter coupling is a major asset, for instance, in spin-physics experiments relying on the simultaneous coupling of several optical transitions. ${ }^{15}$ While microcavities enable near-unity coupling efficiency, the operable spectral window is limited to the narrow linewidth of the cavity. Multimode waveguides support broadband operation, but the coupling efficiency is limited to $<90 \%$. In contrast, PCWs enable broadband nearunity coupling as well as Purcell enhancement of the QD coupled to the waveguide mode. PCWs are composed of air holes etched in a thin membrane (see Figure 1a), resulting in proximity of etched surfaces to the QDs. The proximity of etched surfaces leads to an increase in the charge noise, which has been thoroughly investigated in QDs without electrical contacts. $^{16-19}$ In particular, noncontacted QDs embedded in PCWs exhibited line broadening of $>4 \times$ the natural linewidth, ${ }^{20}$ which may be detrimental for the single-photon source efficiency. Therefore, obtaining transform-limited emission in PCWs is a challenging task and requires low-

Received: May 8, 2020

Published: August 4, 2020 
(a)

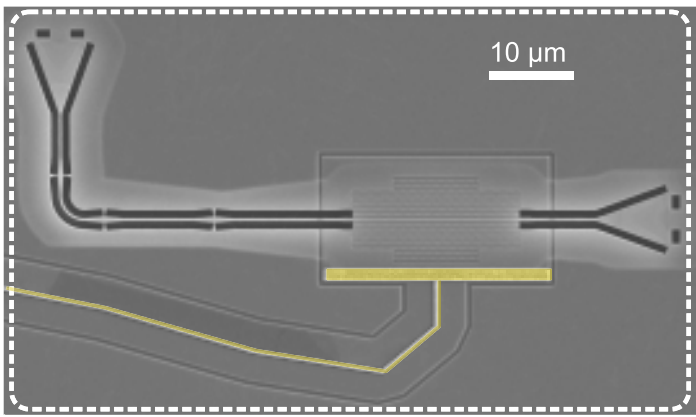

(b)

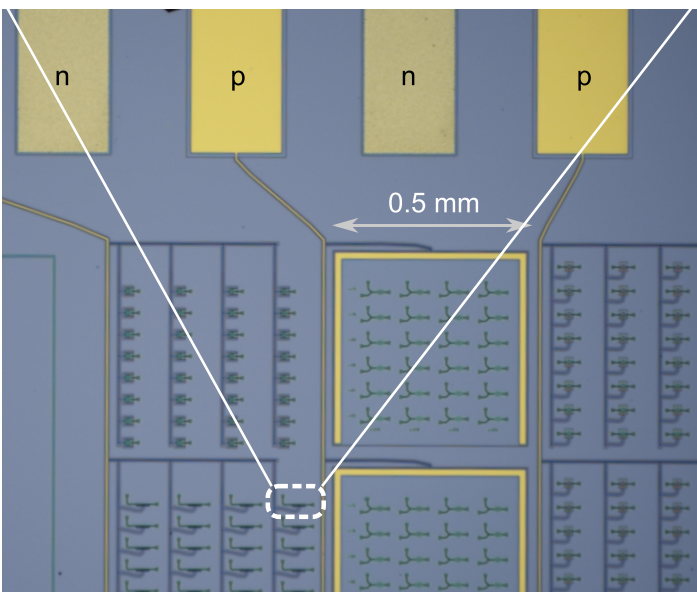

(c)

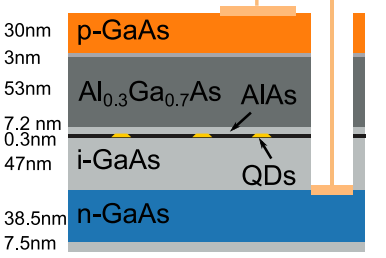

$7.5 \mathrm{~nm}$

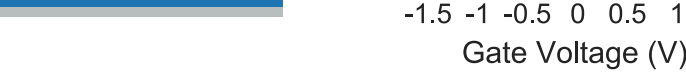

Figure 1. (a) Scanning electron microscope image of a single device with contacts and isolation trenches. (b) Optical microscope image of the fabricated device showing the arrangement of devices in individual groups connected to isolated $p$-type contacts. (c) Layout of the membrane with an embedded $p$-i-n diode heterostructure. (d) Current-voltage $(I-V)$ characteristic of the $p$-i-n diode measured at $T=1.6 \mathrm{~K}$ (circles). The solid curve is a plot of an ideal diode in series with a $R_{\mathrm{s}}=7 \mathrm{k} \Omega$ resistor and with a finite parallel resistance of $10 \mathrm{G} \Omega$.

noise heterostructure semiconductor material and careful nanofabrication, in addition to high-quality electrical contacts.

In this work we establish that electrically contacted QDs overcome charge noise and enable near transform-limited linewidth in a PCW. Through fabrication of high-quality local electrical contacts, near-ideal diode operation was observed with a short RC time constant of $<1 \mu$ s. Linewidths of 79 QDs with resonance frequencies distributed over $6 \mathrm{~nm}$ were measured using resonant transmission (RT) of a weak coherent state and a selection of them compared against the natural linewidth. We observe that at least $65 \%$ of the QDs coupled to the PCWs exhibit near transform-limited lineshapes.

\section{DEVICE FABRICATION AND ELECTRICAL CHARACTERIZATION}

A scanning electron microscope (SEM) image of a typical device from the nanofabricated sample is shown in Figure 1a.
The device consists of a PCW terminated with shallow-etched grating outcouplers, fabricated in a $170 \mathrm{~nm}$ thin suspended gallium arsenide (GaAs) membrane as follows. The membrane is grown using molecular beam epitaxy on a (100) GaAs substrate. The substrate is prepared for growth using an AlAs/ GaAs superlattice followed by a $1150 \mathrm{~nm}$ thick $\mathrm{Al}_{0.75} \mathrm{Ga}_{0.25} \mathrm{As}$ sacrificial layer. A layout of the $170 \mathrm{~nm}$ thick GaAs membrane containing a layer of self-assembled InAs QDs grown on top of the sacrificial layer, is shown in Figure 1c. The QDs are located at the center of the membrane, which ensures maximal coupling of the QD emission to the transverse electric (TE) modes of the waveguide. The sample employed in the measurements has an approximate $\mathrm{QD}$ density of $\approx 10 / \mu \mathrm{m}^{2}$, which results in $\approx 60$ QDs positioned in a PCW of length 20 $\mu \mathrm{m}$. The membrane comprises an ultrathin $p-i-n$ heterostructure diode with the layout shown in Figure 1c, which is used to apply an electric field across the QDs. The electric field helps to reduce the charge noise and allows to tune the QD emission wavelength via the Stark effect. The $n$-type region is located $47 \mathrm{~nm}$ below the QDs to suppress cotunneling and at the same time stabilize the QDs charge state by Coulomb blockade. ${ }^{21}$ A monolayer of AlAs capping of the QDs removes the electron wetting layer states. ${ }^{22}$ A $53 \mathrm{~nm}$ thick $\mathrm{Al}_{0.3} \mathrm{Ga}_{0.7} \mathrm{As}$ layer above the QDs is used as a blocking barrier to limit the current to a few $\mathrm{nA}$ at a bias voltage of $\approx 1 \mathrm{~V}$, where the QDs can be charged with a single electron.

Reactive-ion etching (RIE) in a $\mathrm{BCl}_{3} / \mathrm{Ar}$ chemistry is used to open vias to the $n$-layer. The $\mathrm{Ni} / \mathrm{Ge} / \mathrm{Au} / \mathrm{Ni} / \mathrm{Au}$ contacts are fabricated using electron-beam physical vapor deposition followed by annealing at $430{ }^{\circ} \mathrm{C}$. To establish Ohmic $p$-type contacts, $\mathrm{Cr} / \mathrm{Au}$ contacts are deposited on the surface without further annealing. The shallow-etched grating couplers are patterned by electron-beam lithography (Elionix F-125, acceleration voltage of $125 \mathrm{keV}$ ) and then etched using RIE to a depth of approximately $50 \mathrm{~nm} .^{23}$ The PCWs are fabricated with the process described in ref 24 , followed by hydrofluoric acid undercut to create suspended waveguides.

The full processed chip has a size of $3 \mathrm{~mm} \times 3 \mathrm{~mm}$ and is divided into five sections with physical dimensions of $0.5 \mathrm{~mm}$ $\times 3 \mathrm{~mm}$ each. An optical image displaying some of these sections is shown in Figure $1 \mathrm{~b}$. Each section is connected to separate pairs of electrical contacts, also visible in the image. This design reduces the number of defects or thread dislocations on each diode (i.e., each section), thereby reducing the leakage current. The $n$-doped layer is used as a common ground plane for all devices, while $p$-doped layers and metal wires are used to distribute the voltage uniformly to several devices in parallel. In order to achieve minimum crosstalk between the different sections, an isolation trench with a width of $1 \mu \mathrm{m}$ is patterned around the $p$-contacts and etched with RIE together with the shallow-etched gratings. Some sections are designed with local electrical contacts, such that the field can be applied to a single device. By bringing the contacts close to the QDs and introducing isolation trenches, the capacitance $C$ and the sheet resistance $R$ of the diodes are significantly reduced. This reduction in the contacted area shortens the RC response time of the device and allows for fast operation of the diode. The local electrical contact highlighted in the SEM image in Figure 1a enables individual control of multiple devices on the same chip, which is crucial, for example, for scaling up to interfering multiple emitters. ${ }^{25,26}$

The sample was cooled to $1.6 \mathrm{~K}$ in a closed-cycle helium cryostat with optical and electrical access for QD spectroscopy 
measurements. The different sections on the sample were wired to independent twisted pair transmission lines in the cryostat. As the $n$-contacts are not isolated on the sample, we connect them to the common ground of a multichannel lownoise voltage source $\left(V_{\text {rms }}<1 \mu \mathrm{V}\right)$. This removes potential ground loops caused by any variations in the parasitic resistance on the sample or the transmission lines. We typically measure an RMS voltage noise of $<200 \mu \mathrm{V}$ up to a bandwidth of $10 \mathrm{MHz}$ on the sample transmission lines. This was observed to be limited mostly by the ambient noise picked up by the twisted pair lines. In another cryostat employing coaxial lines, RMS voltage noise of $<80 \mu \mathrm{V}$ has been measured on a similar sample.

The current-voltage $(I-V)$ curve recorded using a sourcemeter is shown in the Figure $1 \mathrm{~d}$. A clear diode turnon at gate voltage $V_{\mathrm{g}}>0.7 \mathrm{~V}$ is observed with very low leakage current. The sample exhibits a near-ideal $I-V$ curve for a $p-i-n$ diode, with the leakage current limited by the source meter noise in the reverse bias. In the Coulomb blockade regime for QD neutral excitons $\left(V_{\mathrm{g}}<1.28 \mathrm{~V}\right)$, the leakage current across the diode is $<1 \mathrm{nA}$, and has thus excellent $I-V$ properties.

\section{RESONANT LINEWIDTH MEASUREMENTS}

A schematic of the laser transmission experiment in the PCW is shown in Figure 2a. The PCWs used in our measurements

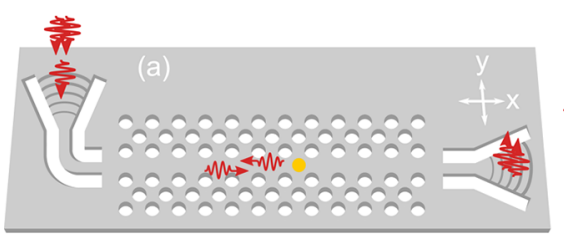

(c)
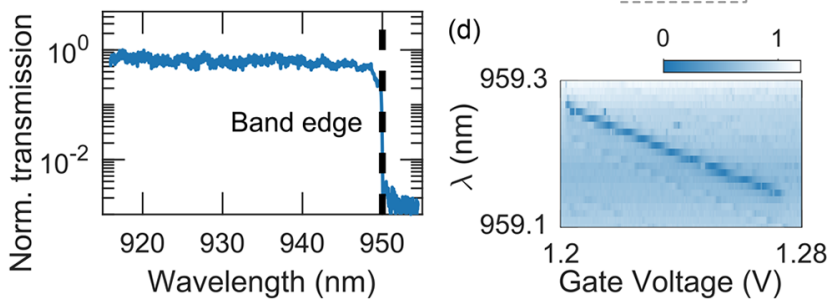

Figure 2. (a) Schematic of the photonic crystal waveguide device employed in the measurements with lattice constant $a=248 \mathrm{~nm}$ and hole radius $r=70 \mathrm{~nm}$. The photonic crystal waveguides are terminated with high-efficiency shallow-etched grating couplers, where the left grating incouples the laser and the right outcouples the transmitted signal. The QD is illustrated with a yellow dot, where a resonant photon is reflected in an RT measurement. (b) Schematic of the optical setup used for transmission measurements. (c) Frequency-dependent laser transmission through the photonic crystal waveguide without QDs, normalized to transmission through a nanobeam waveguide. (d) Charge plateau of a QD neutral exciton coupled to the PCW observed in the resonant transmission of an attenuated narrow-linewidth laser.

have a lattice constant of $a=248 \mathrm{~nm}$ and hole radii $r=70 \mathrm{~nm}$. The PCW is mode-matched to a section with nanobeam waveguide at both ends and terminated with high-efficiency shallow-etched grating couplers ${ }^{23}$ for in- and out-coupling of light. Light is launched into the waveguide from the left, and light transmitted through the PCW is collected on the right grating. In Figure 2b, a schematic of the optical setup is shown, where a tunable narrow-band laser (bandwidth $<1 \mathrm{MHz}$ ) is collimated and imaged to the back focal plane of a wide-field microscope objective. The objective focuses the laser to a spot size which is mode-matched with the in-coupling grating. The light transmitted through the PCW is collected from the right grating using the same microscope objective. The incident laser and collected transmission are separated into different spatial modes using a 5:95 (reflection/transmission) beam splitter, where the transmission arm is used for collection. The collected signal is detected using a superconducting nanowire single-photon detector (SNSPD). The frequency-dependent transmission of the laser through the device at $V_{\mathrm{g}}=1.0 \mathrm{~V}$, where no $\mathrm{QD}$ states are populated due to the high built-in electric field, is shown in Figure 2c. The transmission spectrum is normalized to the transmission through a nanobeam waveguide terminated with identical grating out-couplers, to factor out the frequency-dependent diffraction efficiency of the grating out-couplers. A steep cutoff in the transmittance of $>2$ orders of magnitude is observed at wavelengths longer than $950.2 \mathrm{~nm}$, which corresponds to the band gap of the photonic crystal. This large suppression together with a nearly constant transmission below the cutoff wavelength highlight the excellent photonic properties of the nanofabricated PCWs.

QDs that are efficiently coupled to the PCW exhibit a singlephoton nonlinearity, where the single-photon component of a weak laser resonant with a $\mathrm{QD}$ transition is reflected as illustrated in Figure $2 a^{20,27}$ If the incident photon is detuned from the $\mathrm{QD}$ resonance, the interaction with the $\mathrm{QD}$ vanishes and the photon is transmitted. This resonant scattering appears as a dip in the frequency-dependent transmission across the PCW. Figure $2 \mathrm{~d}$ displays such a dip from an RT measurement of a QD while tuning the applied bias voltage. The charge plateau shows the distinct Coulomb blockade regime for the neutral exciton. Importantly, the charging of the QD occurs at a gate voltage close to the predicted value from bandstructure simulations in contrast to earlier reports. ${ }^{28}$ This agreement is a consequence of the low contact resistance of the sample.

The width of the RT dip, at powers well below the saturation power for the $\mathrm{QD}$, is a reliable measurement of the QD linewidth. ${ }^{27}$ Any charge noise causing the energy levels to shift or fluctuate will degrade the photon-emitter interactions and result in a broadening of the measured linewidth. The incident narrow-bandwidth laser power was attenuated to $P=$ $0.4 \mathrm{pW}$ in the waveguide, which was found to be $<1 \%$ of the saturation power of the QDs. At a gate voltage of $V_{\mathrm{g}}=1.24 \mathrm{~V}$, which corresponds to populating the neutral exciton, the wavelength of the laser is scanned from 944 to $950 \mathrm{~nm}$. The laser wavelength was locked using a wavemeter with a resolution of $50 \mathrm{MHz}(0.15 \mathrm{pm})$. The $6 \mathrm{~nm}$ wavelength range near the cutoff was chosen to capture the slow-light regime of light transport in the PCW, which leads to a Purcell enhancement in the radiative decay rate of the QD. ${ }^{29}$ Several QD resonances, identified as RT dips, were recorded in a single continuous wavelength scan, spanning the whole bandwidth of $6 \mathrm{~nm}$, with a step size of $100 \mathrm{MHz}$, and each RT dip was independently fitted using the model described in ref 20. One such RT dip measured from a QD is shown in Figure $3 \mathrm{a}$, together with the fit to the model. The asymmetry in the number of sampling points on the positive and negative frequency detuning of the RT resonance is due to the Fano line shape, which results in a steeper slope on the positive frequency side. The Fano line shape of the RT dip originates from the interference between the QD resonance and a weak reflection from the mode adapters between PCW and the 


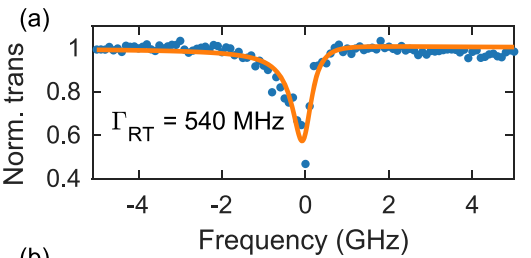

(b)

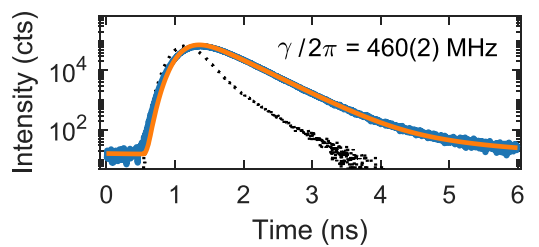

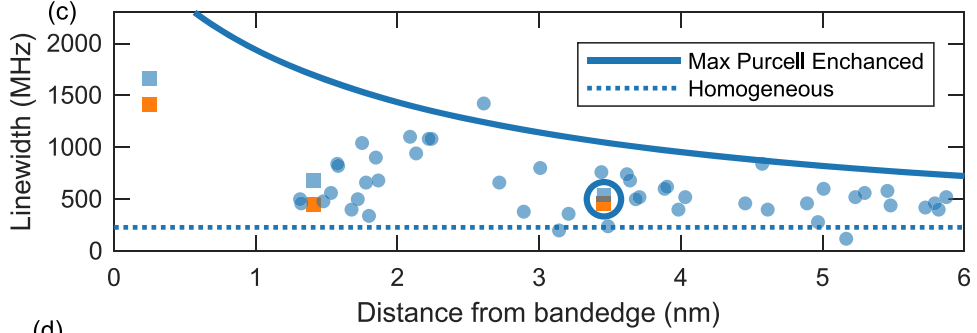

(d) Resonances Fitted res

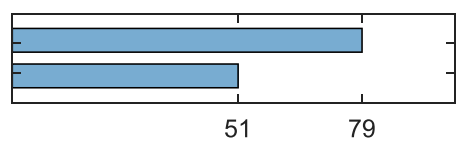

Number of resoances $(\mathrm{e})$

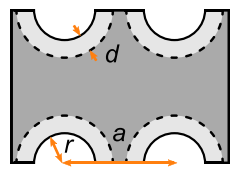

Figure 3. (a) RT line fit of a QD in the photonic crystal waveguide. The fwhm of the fitted linewidth is displayed on the plot. (b) Lifetime of the resonantly excited QD exhibiting a single exponential decay. The black dotted curve is the instrument response function (IRF) of the measurement setup. The single-exponential fit to the data includes the convolution of the model with the IRF. (c) Blue points are the fwhm of fitted RT linewidths similar to (a) for 51 resonances of QDs in the photonic crystal waveguide. Orange squares are transform limited linewidths of three of the QD, extracted from the decay rate of lifetime measurements similar to (b). The corresponding RT linewidth are also plotted with a square (blue) marker. The encircled dots are the measurements shown in (a) and (b). Solid blue line is the maximum achievable Purcell enhanced linewidth given the homogeneous linewidth shown with a dotted line. (d) Overview of the total number of QD resonances found in the RT measurement and the number of QD resonances that had a pronounced RT dip whose linewidth could be extracted. (e) Photonic crystal waveguide with a hole radius $r$ and lattice constant $a$. The lighter gray area at distance $d$ from the holes illustrates the region where the QDs are affected by surface charges.

nanobeam waveguides in the device. The linewidth of the QD resonance $\Gamma_{\mathrm{RT}}$ is extracted as the full-width at half-maximum (fwhm) of the fitted curve, which allows to reach values very close to (or even below) the data sampling resolution. The narrowest extracted linewidths may be less precise due to the limited number of data points. For simplicity, the fwhm of the fitted curve is extracted with the Fano parameter omitted (i.e., symmetric line shape). This approach leads to upper-bound estimates of the linewidth.

For comparison, the transform-limited linewidth $\Gamma$ is extracted from a time-resolved resonance fluorescence (RF) measurement of the QD under pulsed resonant excitation. In this configuration, the $\mathrm{QD}$ is excited with a pulsed laser (repetition rate $=72.6 \mathrm{MHz}$; pulse length $\approx 10 \mathrm{ps}$ ). The photons emitted by the QD couple to the propagating PCW mode and are collected at the grating as before. The spatial separation between the QD (excitation laser beam position) and the collection grating ensures a large suppression of the excitation laser in the collection. The RF measurement involves precisely locating the QDs and suppressing the resonant laser scatter, which is challenging and timeconsuming. Hence, the time-resolved measurements were carried out only on three of the QDs at distinct frequencies. Furthermore, time-resolved measurements were also carried out for a few QDs outside the nanostructures (i.e., in bulk) to estimate the homogeneous linewidth of the QDs. Figure $3 \mathrm{~b}$ shows the time-resolved resonance fluorescence of the QD whose RT measurement is shown in Figure 3a. The data is modeled with a single-exponential decay convolved with the instrument response function to extract the radiative decay rate $\gamma$. The extracted $\gamma$ is used to estimate the transform-limited linewidth $\Gamma=\gamma / 2 \pi=460 \mathrm{MHz}$. This estimate of $\Gamma$ may be considered a lower bound, since any additional nonradiative processes would increase it; however, nonradiative recombination was found to be negligible in photonic-crystal membranes in a previous work. ${ }^{30}$ Comparing the natural linewidth against $\Gamma_{\mathrm{RT}}$, we extract that $\Gamma_{\mathrm{RT}} \leq 1.18 \Gamma$, which demonstrates that the noise affecting this specific QD is strongly suppressed. The residual broadening could be attributed to a slow spectral diffusion of the QD resonances (typical time scale of $>5 \mathrm{~ms}$ in our sample) and nuclear spin noise. $^{10}$

The RT linewidths of the QDs are plotted against their spectral location with respect to the bandedge (waveguide cutoff) in Figure 3c with blue-filled markers. The transformlimited linewidths estimated from RF measurements are plotted as orange squares, with their corresponding RT linewidth also indicated with squares. The QD whose data is shown in Figure $3 \mathrm{a}, \mathrm{b}$ is marked with an open circle. Only one QD very close to the bandedge was analyzed due to the difficulty of fitting the RT dips on the steeply rising bandedge. The measured RT dips cover a large range of wavelengths, demonstrating the large bandwidth performance of the PCW. The observed variation in the measured linewidths (120-1660 $\mathrm{MHz}$ ) across the QDs is a consequence of the wavelength and spatial position dependence of the Purcell factor in a PCW. ${ }^{29}$ Using the radiative decay rate measurements of QDs outside the nanostructure, we estimate the average homogeneous linewidth to be $\Gamma_{\text {hom }} \approx 230 \pm 40 \mathrm{MHz}$ (dashed line in Figure $3 c$ ). The Purcell factor at a specific wavelength is sensitive to the QD dipole orientation and location within the PCW. The wavelength-dependence of the maximum Purcell enhanced linewidth is extracted from numerical calculations, and plotted as the solid curve in Figure 3c. We observe that the maximum Purcell enhanced linewidth follows the measured $\Gamma_{\mathrm{RT}}$ at all wavelengths as an upper-bound. Few $\mathrm{QDs}$ exhibit $\Gamma_{\mathrm{RT}}$ below the homogeneous linewidth, which indicates suppression of the radiative decay rate, and remarkably narrow linewidths are achieved. This observation is also consistent with the PCW's ability to suppress decay rates depending on the QD dipoles location and orientation. The variation of the RT linewidths as a function of distance from the bandedge is therefore explained by the Purcell factor dependence on the dipole orientation and the location of the QDs. For three QDs at spatially and spectrally random locations, we measure the natural linewidth and observe $\Gamma_{\mathrm{RT}} / \Gamma=1.17,1.51$, and 1.18 , respectively, that 
highlights the excellent charge noise suppression. These three representative measurements together with the observation that $\Gamma_{\mathrm{RT}}$ over 51 QDs is bounded by the maximum Purcellenhanced linewidth indicates broadband transform-limited operation of the QDs in PCWs.

A total of 79 QD resonances were found in the frequency scan on two devices, of which 51 (i.e., 65\%) were modeled to extract $\Gamma_{\mathrm{RT}}$, as summarized in Figure $3 \mathrm{~d}$. The linewidths of the remaining 28 QDs were not analyzed due to one of the two factors: (i) very shallow RT dip that could not be robustly fitted or (ii) noisy and spectrally broad RT dip that was affected by slow time scale spectral diffusion. Since the QDs are randomly distributed in the PCW, a selection of them would be weakly coupled to the waveguide mode, either due to their spatial position or the orientation of the dipole. Such QDs exhibit a shallow RT dip due to the weak coupling to the PCW.

We estimate an approximate upper-bound on the distance $d$ from the etched surfaces, beyond which the QDs exhibit a transform-limited line shape as follows. QDs located near an etched surface experience spectral diffusion of the QD resonance due to the fluctuating electric fields caused by charge traps on the surface. This spectral diffusion typically occurs on a time scale of a few milliseconds and is a random process. Therefore, the measured averaged line shape (over a few seconds) will deviate from the Lorentzian line shape due to the Gaussian broadening. ${ }^{19}$ Hence, we can assume that the fraction of the QD resonances that are not modeled by a Lorentzian to be a robust indicator for the fraction of QDs located within the distance $d$. The total area in a unit cell of the PCW is $A_{\text {total }}$ (total gray shaded region in Figure $3 \mathrm{c}$ ). We then define $A_{\lim }$ as the area of the PCW where QDs exhibit a near transform-limited linewidth (dark gray area bounded by the dotted circles with distance $d$ to the air holes). Then, by equating the ratio of the areas to the fraction of fitted QDs $f=$ $51 / 79=A_{\text {lim }} / A_{\text {total }}$, the limiting distance is estimated to be $d<$ $43 \mathrm{~nm}$.

This indicates that a semiconductor heterostructure, together with the high-quality electrical contacts, achieves optimal operation of the QDs close to the center of the PCW, where efficient coupling to the guided mode is expected. ${ }^{8}$

\section{ELECTRICAL SWITCHING OF QUANTUM DOTS}

Alongside the noise-free operation of QDs, the near-ideal $p$-i-n diode $I-V$ curve with low contact resistance indicates a short $\mathrm{RC}$ time constant, which can enable fast electrical switching and control of the QD resonances. ${ }^{31}$ We measure the switching time using an RF experiment, where the $\mathrm{QD}$ is excited by a narrow-bandwidth continuous-wave laser. The gate voltage $V_{\mathrm{g}}$ across the QD is sinusoidally modulated around the resonant voltage of the QD. The modulation tunes the QD in and out of resonance with the excitation laser, which in turn modulates the fluorescence intensity. Experimentally, we employ a biastee to mix a DC and an AC voltage source, with the DC offset $V_{\mathrm{DC}}$ set to the resonant voltage of the $\mathrm{QD}$ and the peak-topeak AC amplitude $V_{\mathrm{AC}}=100 \mathrm{mV}$, as illustrated in the inset of Figure 4.

Instead of measuring the modulation in the fluorescence intensity that requires a high-frequency lock-in amplifier, we measure the time-averaged fluorescence (integration time of 1 s). The measured fluorescence intensity with increasing frequency of the $\mathrm{AC}$ modulation $f_{\mathrm{AC}}$ from $100 \mathrm{~Hz}$ to 60 $\mathrm{MHz}$ is shown in Figure 4. As the voltage linewidth of the QD

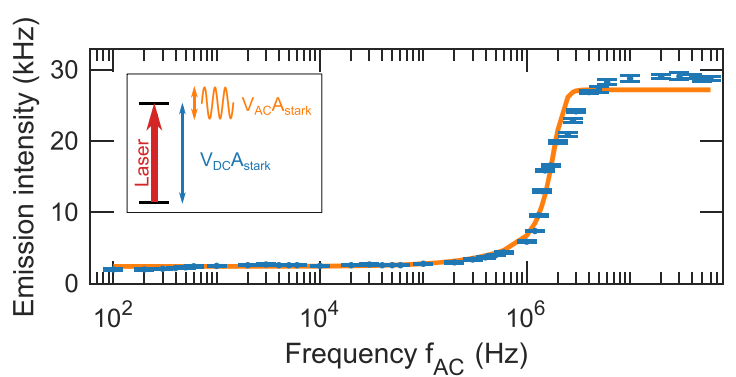

Figure 4. Measurement of the RC constant. Blue points are the cycleaveraged resonance fluorescence from a QD while sinusoidally modulating the bias voltage across the $p-i-n$ diode at various frequencies $f_{\mathrm{AC}}$. The orange curve is a fit to the data, which yields an RC time constant $\tau_{\mathrm{RC}}=0.4 \mu \mathrm{s}$.

is $1 \mathrm{mV}$, which is very small in comparison to $V_{\mathrm{AC}}$, the $\mathrm{QD}$ is resonant with the laser for a very small fraction of the time per cycle. This results in low emission intensity at small $f_{\mathrm{AC}}$. As $f_{\mathrm{AC}}$ is increased close the RC time constant of the diode, the amplitude of modulation experienced by the QD decreases, which increases the emission intensity. We observe that, around $f_{\mathrm{AC}}=3 \mathrm{MHz}$, the Stark tuning of the $\mathrm{QD}$ cannot follow the $V_{\mathrm{AC}}$ and the emission intensity saturates to the unmodulated value. The cycle-averaged $\mathrm{QD}$ fluorescence intensity $I_{\mathrm{QD}}\left(f_{\mathrm{AC}}\right)$ is modeled as

$$
I_{\mathrm{QD}}\left(f_{\mathrm{AC}}\right)=I_{0} \int_{-A\left(f_{\mathrm{AC}}\right)}^{A\left(f_{\mathrm{AC}}\right)} S\left(V-V_{\mathrm{DC}}\right) \mathrm{d} V
$$

where $A\left(f_{\mathrm{AC}}\right) \equiv\left(V_{\mathrm{AC}} / 2\right) \exp \left[-2 \pi f_{\mathrm{AC}} \tau_{\mathrm{RC}}\right], S\left(V-V_{\mathrm{DC}}\right)$ is the measured voltage response of the $\mathrm{QD}$, and $I_{0}$ is the measured resonance fluorescence intensity without modulation. The model yields an RC time constant $\tau_{\mathrm{RC}}=0.4 \mu \mathrm{s}$, that is, a cutoff frequency of $1 /\left(2 \pi \tau_{\mathrm{RC}}\right)=3.98 \mathrm{MHz}$ for the experimental data. The measured $\tau_{\mathrm{RC}}$ together with the $7 \mathrm{k} \Omega$ series resistance estimated from the $I-V$ curve results in a capacitance $C=57$ $\mathrm{pF}$, which is close to the expected value for the contacted area. $^{32}$ The switching speed can be further increased by reducing the contacted area, which in the current configuration covers a full section $(0.5 \mathrm{~mm} \times 3 \mathrm{~mm})$ where all the devices share the same pair of contacts. By dedicating a contact pair to a single PCW with a total area of around $500 \mu \mathrm{m}^{2}$, switching speeds of $\mathrm{GHz}$ could be reached. ${ }^{33}$

\section{CONCLUSION}

In summary, we have demonstrated that charge noise in QDs coupled to PCWs can be largely overcome by employing electrical-contacted QD heterostructures. Statistics on several QDs reveal that $65 \%$ of the QDs exhibit near transform-limited linewidths, which highlight the possibility to realize scalable single-photon sources for quantum information processing. ${ }^{34}$ While some residual surface-induced noise persists, the region of influence of this noise is limited to $<43 \mathrm{~nm}$ from etched surfaces and could be potentially overcome either through surface passivation techniques ${ }^{35,36}$ or through deterministic positioning of QDs. ${ }^{37-39}$ High-speed operation of the $p-i-n$ diode with a cutoff frequency of $\approx 4 \mathrm{MHz}$ is demonstrated by measuring the fluorescence from a voltage-modulated QD. The short RC time constant in combination with near transformlimited linewidths of several QDs paves the way for multiemitter based quantum information processing protocols, which will greatly benefit from the independent and 
deterministic control of individual transform-limited emitters. $^{40-42}$

\section{AUTHOR INFORMATION}

\section{Corresponding Authors}

Ravitej Uppu - Center for Hybrid Quantum Networks (Hy-Q),

Niels Bohr Institute, University of Copenhagen, DK-2100

Copenhagen, Denmark; @ orcid.org/0000-0002-8052-9427;

Email: ravitej.uppu@nbi.ku.dk

Peter Lodahl - Center for Hybrid Quantum Networks (Hy-Q), Niels Bohr Institute, University of Copenhagen, DK-2100 Copenhagen, Denmark; Email: lodahl@nbi.ku.dk

\section{Authors}

Freja T. Pedersen - Center for Hybrid Quantum Networks (Hy-Q), Niels Bohr Institute, University of Copenhagen, DK2100 Copenhagen, Denmark; 이이.org/0000-0001-83513474

Ying Wang - Center for Hybrid Quantum Networks (Hy-Q), Niels Bohr Institute, University of Copenhagen, DK-2100 Copenhagen, Denmark

Cecilie T. Olesen - Center for Hybrid Quantum Networks (Hy-Q), Niels Bohr Institute, University of Copenhagen, DK2100 Copenhagen, Denmark

Sven Scholz - Lehrstuhl für Angewandte Festkörperphysik, Ruhr-Universität Bochum, D-44780 Bochum, Germany

Andreas D. Wieck - Lehrstuhl für Angewandte

Festkörperphysik, Ruhr-Universität Bochum, D-44780 Bochum, Germany

Arne Ludwig - Lehrstuhl für Angewandte Festkörperphysik, Ruhr-Universität Bochum, D-44780 Bochum, Germany

Matthias C. Löbl - Department of Physics, University of Basel, CH-4056 Basel, Switzerland

Richard J. Warburton - Department of Physics, University of Basel, CH-4056 Basel, Switzerland

Leonardo Midolo - Center for Hybrid Quantum Networks (Hy-Q), Niels Bohr Institute, University of Copenhagen, DK2100 Copenhagen, Denmark

Complete contact information is available at:

https://pubs.acs.org/10.1021/acsphotonics.0c00758

\section{Notes}

The authors declare the following competing financial interest(s): P.L. is founder and minority shareholder in the company Sparrow Quantum.

\section{ACKNOWLEDGMENTS}

We gratefully acknowledge financial support from Danmarks Grundforskningsfond (DNRF; Center for Hybrid Quantum Networks (Hy-Q, DNRF139)), H2020 European Research Council (ERC; SCALE), Styrelsen for Forskning og Innovation (FI; 5072-00016B QUANTECH), Bundesministerium für Bildung und Forschung (BMBF; 16KIS0867, Q.Link.X), Deutsche Forschungsgemeinschaft (DFG; TRR 160), SNF (Project No. 200020 156637), and NCCR QSIT.

\section{REFERENCES}

(1) Kimble, H. J. The quantum internet. Nature 2008, 453, 10231030.

(2) Melet, R.; Voliotis, V.; Enderlin, A.; Roditchev, D.; Wang, X.; Guillet, T.; Grousson, R. Resonant excitonic emission of a single quantum dot in the Rabi regime. Phys. Rev. B: Condens. Matter Mater. Phys. 2008, 78, 073301.
(3) Lodahl, P.; Mahmoodian, S.; Stobbe, S. Interfacing single photons and single quantum dots with photonic nanostructures. Rev. Mod. Phys. 2015, 87, 347-400.

(4) Somaschi, N.; Giesz, V.; De Santis, L.; Loredo, J. C.; Almeida, M. P.; Hornecker, G.; Portalupi, S. L.; Grange, T.; Anton, C.; Demory, J.; Gomez, C.; Sagnes, I.; Lanzillotti-Kimura, N. D.; Lemaitre, A.; Auffeves, A.; White, A. G.; Lanco, L.; Senellart, P. Near-optimal single-photon sources in the solid state. Nat. Photonics 2016, 10, 340 .

(5) Liu, F.; Brash, A. J.; O'Hara, J.; Martins, L. M.; Phillips, C. L.; Coles, R. J.; Royall, B.; Clarke, E.; Bentham, C.; Prtljaga, N.; Itskevich, I. E.; Wilson, L. R.; Skolnick, M. S.; Fox, A. M. High Purcell factor generation of indistinguishable on-chip single photons. Nat. Nanotechnol. 2018, 13, 835-840.

(6) Hepp, S.; Jetter, M.; Portalupi, S. L.; Michler, P. Semiconductor Quantum Dots for Integrated Quantum Photonics. Adv. Quantum Technol. 2019, 2, 1900020.

(7) Anderson, M.; Müller, T.; Huwer, J.; Skiba-Szymanska, J.; Krysa, A.; Stevenson, R.; Heffernan, J.; Ritchie, D.; Shields, A. Quantum teleportation using highly coherent emission from telecom C-band quantum dots. npj Quantum Inf. 2020, 6, 1-7.

(8) Arcari, M.; Söllner, I.; Javadi, A.; Lindskov Hansen, S.; Mahmoodian, S.; Liu, J.; Thyrrestrup, H.; Lee, E. H.; Song, J. D.; Stobbe, S.; Lodahl, P. Near-Unity Coupling Efficiency of a Quantum Emitter to a Photonic Crystal Waveguide. Phys. Rev. Lett. 2014, 113, No. 093603.

(9) Wang, H.; Duan, Z.-C.; Li, Y.-H.; Chen, S.; Li, J.-P.; He, Y.-M.; Chen, M.-C.; He, Y.; Ding, X.; Peng, C.-Z.; Schneider, C.; Kamp, M.; Höfling, S.; Lu, C.-Y.; Pan, J.-W. Near-Transform-Limited Single Photons from an Efficient Solid-State Quantum Emitter. Phys. Rev. Lett. 2016, 116, 213601.

(10) Kuhlmann, A. V.; Houel, J.; Ludwig, A.; Greuter, L.; Reuter, D.; Wieck, A. D.; Poggio, M.; Warburton, R. J. Charge noise and spin noise in a semiconductor quantum device. Nat. Phys. 2013, 9, 570575.

(11) Warburton, R. J. Single spins in self-assembled quantum dots. Nat. Mater. 2013, 12, 483-493.

(12) Kuhlmann, A. V.; Prechtel, J. H.; Houel, J.; Ludwig, A.; Reuter, D.; Wieck, A. D.; Warburton, R. J. Transform-limited single photons from a single quantum dot. Nat. Commun. 2015, 6, 8204.

(13) Najer, D.; Söllner, I.; Sekatski, P.; Dolique, V.; Löbl, M. C.; Riedel, D.; Schott, R.; Starosielec, S.; Valentin, S. R.; Wieck, A. D.; Sangouard, N.; Ludwig, A.; Warburton, R. J. A gated quantum dot strongly coupled to an optical microcavity. Nature 2019, 575, 622627.

(14) Thyrrestrup, H.; Kirsanske, G.; Le Jeannic, H.; Pregnolato, T.; Zhai, L.; Raahauge, L.; Midolo, L.; Rotenberg, N.; Javadi, A.; Schott, R.; Wieck, A. D.; Ludwig, A.; Lobl, M. C.; Sollner, I.; Warburton, R. J.; Lodahl, P. Quantum optics with near-lifetime-limited quantum-dot transitions in a nanophotonic waveguide. Nano Lett. 2018, 18, 18011806.

(15) Javadi, A.; Ding, D.; Appel, M. H.; Mahmoodian, S.; Lobl, M. C.; Sollner, I.; Schott, R.; Papon, C.; Pregnolato, T.; Stobbe, S.; Midolo, L.; Schroder, T.; Wieck, A. D.; Ludwig, A.; Warburton, R. J.; Lodahl, P. Spin-photon interface and spin-controlled photon switching in a nanobeam waveguide. Nat. Nanotechnol. 2018, 13, 398-403.

(16) Wang, C. F.; Badolato, A.; Wilson-Rae, I.; Petroff, P. M.; Hu, E.; Urayama, J.; Imamoglu, A. Optical properties of single InAs quantum dots in close proximity to surfaces. Appl. Phys. Lett. 2004, $85,3423-3425$.

(17) Houel, J.; Kuhlmann, A. V.; Greuter, L.; Xue, F.; Poggio, M.; Gerardot, B. D.; Dalgarno, P. A.; Badolato, A.; Petroff, P. M.; Ludwig, A.; Reuter, D.; Wieck, A. D.; Warburton, R. J. Probing Single-Charge Fluctuations at a GaAs/AlAs Interface Using Laser Spectroscopy on a Nearby InGaAs Quantum Dot. Phys. Rev. Lett. 2012, 108, 107401.

(18) Ha, N.; Mano, T.; Chou, Y.-L.; Wu, Y.-N.; Cheng, S.-J.; Bocquel, J.; Koenraad, P. M.; Ohtake, A.; Sakuma, Y.; Sakoda, K.; Kuroda, T. Size-dependent line broadening in the emission spectra of 
single GaAs quantum dots: Impact of surface charge on spectral diffusion. Phys. Rev. B: Condens. Matter Mater. Phys. 2015, 92, 075306.

(19) Liu, J.; Konthasinghe, K.; Davanço, M.; Lawall, J.; Anant, V.; Verma, V.; Mirin, R.; Nam, S. W.; Song, J. D.; Ma, B.; Chen, Z. S.; Ni, H. Q.; Niu, Z. C.; Srinivasan, K. Single Self-Assembled InAs/GaAs Quantum Dots in Photonic Nanostructures: The Role of Nanofabrication. Phys. Rev. Appl. 2018, 9, 064019.

(20) Javadi, A.; Söllner, I.; Arcari, M.; Hansen, S. L.; Midolo, L.; Mahmoodian, S.; Kiršanske, G.; Pregnolato, T.; Lee, E.; Song, J.; Stobbe, S.; Lodahl, P. Single-photon non-linear optics with a quantum dot in a waveguide. Nat. Commun. 2015, 6, 8655.

(21) Drexler, H.; Leonard, D.; Hansen, W.; Kotthaus, J. P.; Petroff, P. M. Spectroscopy of Quantum Levels in Charge-Tunable InGaAs Quantum Dots. Phys. Rev. Lett. 1994, 73, 2252-2255.

(22) Löbl, M. C.; Scholz, S.; Söllner, I.; Ritzmann, J.; Denneulin, T.; Kovács, A.; Kardynał, B. E.; Wieck, A. D.; Ludwig, A.; Warburton, R. J. Excitons in InGaAs quantum dots without electron wetting layer states. Commun. Phys. 2019, 2, 93.

(23) Zhou, X.; Kulkova, I.; Lund-Hansen, T.; Hansen, S. L.; Lodahl, P.; Midolo, L. High-efficiency shallow-etched grating on GaAs membranes for quantum photonic applications. Appl. Phys. Lett. 2018, 113, 251103.

(24) Midolo, L.; Pregnolato, T.; Kiršanskè, G.; Stobbe, S. Soft-mask fabrication of gallium arsenide nanomembranes for integrated quantum photonics. Nanotechnology 2015, 26, 484002.

(25) Zheng, H.; Baranger, H. U. Persistent Quantum Beats and Long-Distance Entanglement from Waveguide-Mediated Interactions. Phys. Rev. Lett. 2013, 110, 113601.

(26) Mahmoodian, S.; Cepulkovskis, M.; Das, S.; Lodahl, P.; Hammerer, K.; Sørensen, A. S. Strongly correlated photon transport in waveguide quantum electrodynamics with weakly coupled emitters. Phys. Rev. Lett. 2018, 121, 143601.

(27) Shen, J.-T.; Fan, S. Coherent photon transport from spontaneous emission in one-dimensional waveguides. Opt. Lett. 2005, 30, 2001-2003.

(28) Pinotsi, D.; Fallahi, P.; Miguel-Sanchez, J.; Imamoglu, A. Resonant spectroscopy on charge tunable quantum dots in photonic crystal structures. IEEE J. Quantum Electron. 2011, 47, 1371-1374.

(29) Javadi, A.; Mahmoodian, S.; Söllner, I.; Lodahl, P. Numerical modeling of the coupling efficiency of single quantum emitters in photonic-crystal waveguides. J. Opt. Soc. Am. B 2018, 35, 514-522.

(30) Wang, Q.; Stobbe, S.; Lodahl, P. Mapping the Local Density of Optical States of a Photonic Crystal with Single Quantum Dots. Phys. Rev. Lett. 2011, 107, 167404.

(31) Prechtel, J. H.; Dalgarno, P. A.; Hadfield, R. H.; McFarlane, J.; Badolato, A.; Petroff, P. M.; Warburton, R. J. Fast electro-optics of a single self-assembled quantum dot in a charge-tunable device. J. Appl. Phys. 2012, 111, 043112.

(32) Midolo, L.; Hansen, S. L.; Zhang, W.; Papon, C.; Schott, R.; Ludwig, A.; Wieck, A. D.; Lodahl, P.; Stobbe, S. Electro-optic routing of photons from a single quantum dot in photonic integrated circuits. Opt. Express 2017, 25, 33514-33526.

(33) Pagliano, F.; Cho, Y.; Xia, T.; Van Otten, F.; Johne, R.; Fiore, A. Dynamically controlling the emission of single excitons in photonic crystal cavities. Nat. Commun. 2014, 5, 5786.

(34) Uppu, R.; Pedersen, F. T.; Wang, Y.; Olesen, C. T.; Papon, C.; Zhou, X.; Midolo, S.; Leonardo, S.; Wieck, A. D.; Ludwig, A.; Lodahl, P. Scalable integrated single-photon source. arXiv:2003.08919 [quantph] 2020, na.

(35) Guha, B.; Marsault, F.; Cadiz, F.; Morgenroth, L.; Ulin, V.; Berkovitz, V.; Lemaitre, A.; Gomez, C.; Amo, A.; Combrié, S.; Gérard, B.; Leo, G.; Favero, I. Surface-enhanced gallium arsenide photonic resonator with quality factor of $6 \times 10^{6}$. Optica 2017, 4, 218-221.

(36) Kuruma, K.; Ota, Y.; Kakuda, M.; Iwamoto, S.; Arakawa, Y. Surface-passivated high-Q GaAs photonic crystal nanocavity with quantum dots. APL Photonics 2020, 5, 046106.

(37) He, Y.-M.; Liu, J.; Maier, S.; Emmerling, M.; Gerhardt, S.; Davanço, M.; Srinivasan, K.; Schneider, C.; Höfling, S. Deterministic implementation of a bright, on-demand single-photon source with near-unity indistinguishability via quantum dot imaging. Optica 2017, 4, 802-808.

(38) Pregnolato, T.; Chu, X.-L.; Schröder, T.; Schott, R.; Wieck, A. D.; Ludwig, A.; Lodahl, P.; Rotenberg, N. Deterministic positioning of quantum dots in nanophotonic waveguides. APL Photonics 2020, 5, 086101 .

(39) Ollivier, H.; Maillette de Buy Wenniger, I.; Thomas, S.; Wein, S. C.; Harouri, A.; Coppola, G.; Hilaire, P.; Millet, C.; Lemaitre, A.; Sagnes, I.; Krebs, O.; Lanco, L.; Loredo, J. C.; Anton, C.; Somaschi, N.; Senellart, P. Reproducibility of High-Performance Quantum Dot Single-Photon Sources. ACS Photonics 2020, 7, 1050-1059.

(40) Hettich, C.; Schmitt, C.; Zitzmann, J.; Kühn, S.; Gerhardt, I.; Sandoghdar, V. Nanometer Resolution and Coherent Optical Dipole Coupling of Two Individual Molecules. Science 2002, 298, 385-389.

(41) Sipahigil, A.; Evans, R. E.; Sukachev, D. D.; Burek, M. J.; Borregaard, J.; Bhaskar, M. K.; Nguyen, C. T.; Pacheco, J. L.; Atikian, H. A.; Meuwly, C.; Camacho, R. M.; Jelezko, F.; Bielejec, E.; Park, H.; Loncar, M.; Lukin, M. D. An integrated diamond nanophotonics platform for quantum-optical networks. Science 2016, 354, 847-850.

(42) Kim, J.-H.; Aghaeimeibodi, S.; Richardson, C. J. K.; Leavitt, R. P.; Waks, E. Super-Radiant Emission from Quantum Dots in a Nanophotonic Waveguide. Nano Lett. 2018, 18, 4734-4740. 